\title{
SLEEP DISORDERED BREATHING AND SMALL VESSEL DISEASE IN STROKE: ACUTE AND SIX MONTHS FOLLOW-UP
}

Sofie A. Simonsen, $M D^{1}$, Adam V. Andersen, MD¹, Anders S. West, MD¹, Frauke Wolfram, MD², Poul Jennum MD, DMSc ${ }^{3}$, Helle K. Iversen MD, DMSc ${ }^{1}$

Rigshospitalet

${ }^{1}$ Clinical Stroke Research Unit, Department of Neurology; '2Department of Radiology;

${ }^{3}$ Danish Center for Sleep Medicine. Rigshospitalet, University of Copenhagen.

\section{BACKGROUND}

Sleep disordered breathing (SDB) occurs frequently after stroke, is associated with poor functional outcome and increased risk of mortality. The aim of this study was to investigate associations between stroke etiology and SDB, as well as SDB development over time.

\section{METHODS}

This observational study included stroke patients within seven days from onset, and re-tested after six months. We performed a polysomnography (photo with permission from the patient) and calculated the apnea hypopnea index $(\mathrm{AHI})$. Sleepiness was scored by Epworth sleepiness scale (ESS) and sleep quality by Pittsburgh sleep quality index (PSQI). The ischemic strokes were classified according to the TOAST classification and a total SVD score (0-4) was calculated

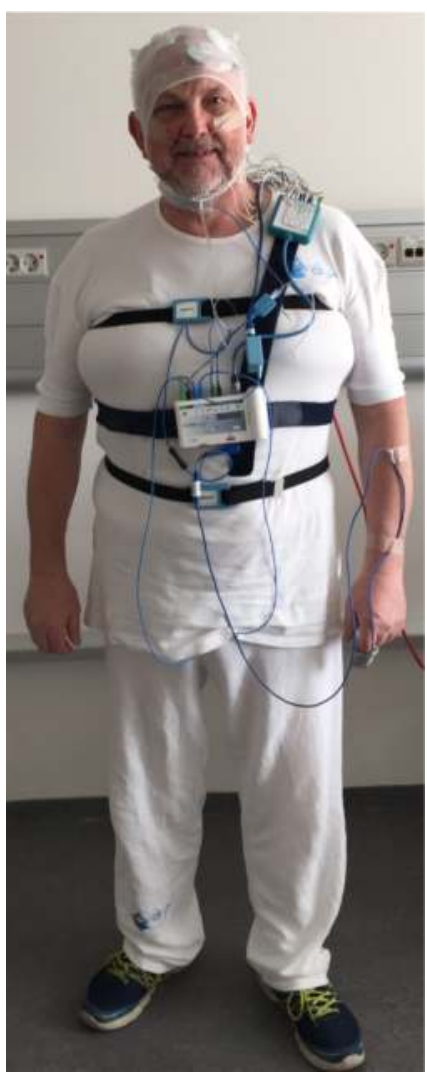
based on MRI.

Figure. Percentage of patients with $\mathrm{AHI} \geq 15$ at both sleep examinations in the five different SVD score groups. A: SVD scores in the acute state, B: SVD scores at follow-up.

\section{CONCLUSION}

- SDB and sleepiness were frequent and improved during the first six month after stroke.

- $\mathrm{AHI}$ and treatment-requiring SDB in both the acute state and follow-up was associated to increased SVD score, but not to the TOAST classification groups.

- The SVD score is recommended used in future studies on SDB in stroke.

- Stroke patients should be tested for SDB.

\section{RESULTS}

- 101 patients were included, median age 68 years (range $36-88$ ), 57\% men.

- 58 patients had follow-up.

- $90 \%$ were ischemic strokes.

- Stroke severity was median NIHSS 2 (range 0-16).

- SDB, defined as $A H I \geq 15$, was found in $57 \%$ of patients in the acute state and in $50 \%$ of patients at follow-up.

- AHI decreased over time, median change 3.9, 95\% confidence interval [95\% Cl]: 0.7-8.3; $p=0.012$.

- Sleepiness decreased over time: ESS $(1.5,95 \% \mathrm{Cl}$ : $0.5-3.0 ; p=0.01)$, but sleep quality was unchanged: PSQI (0.5, 95\%Cl: $1.0-1.5 ; p=0.59)$.

- AHI was not associated with stroke severity (NIHSS), stroke type (ischemic/hemorrhagic), or the TOAST classification subgroups.

- The total SVD score was associated to AHI at follow-up $(p=0.033)$, but not in the acute phase $(p=0.08)$.

- The number of patients with indication for treatment for SDB $(A H I \geq 15)$ at both the acute state and follow-up increased with increased SVD score (Fishers' exact test: $p<0.001$ ).
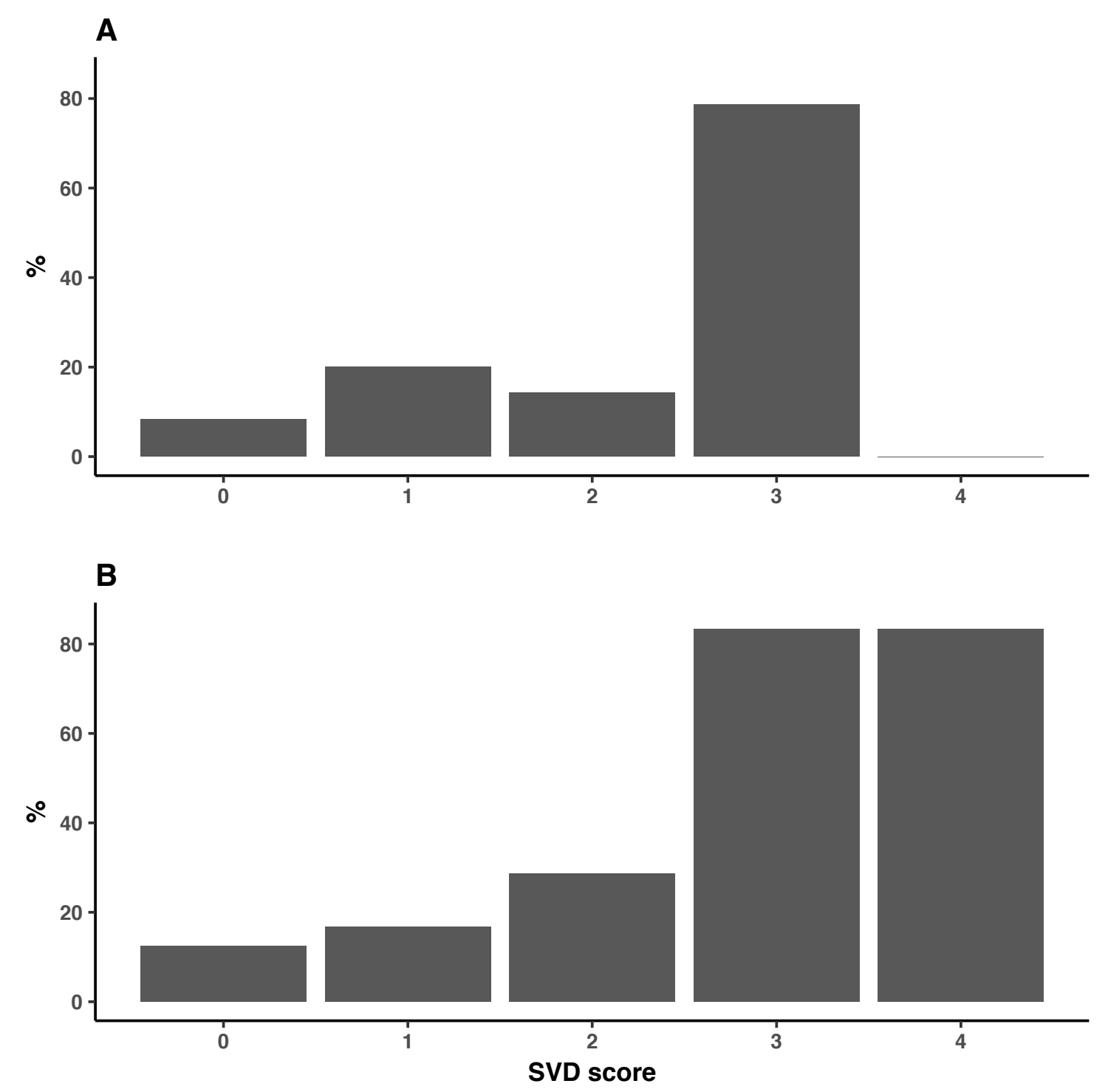